\title{
Hematemesis in Alcoholics: Not Always the Same Problem. A Case Report of Successful Percutaneous Arterial Embolization of Spontaneously Ruptured Splenic Artery Aneurysm Causing Hematemesis, Hematochezia and Hemodynamic Instability
}

\author{
Adam R. Markowski, ${ }^{1,2 *}$,Kazimierz Kordecki ${ }^{3}$, Vitalii Grinevych ${ }^{3}$, Pawet Rogalski ${ }^{4}$ and Andrzej Baniukiewicz ${ }^{4}$ \\ ${ }^{1}$ Department of Internal Medicine and Gastroenterology, Polish Red Cross Memorial Municipal Hospital, 79 Henryk Sienkiewicz Street, 15-003 \\ Bialystok, Poland \\ ${ }^{2}$ Department of Emergency Medicine, Medical University of Bialystok, 24A Maria Sklodowska-Curie Street, 15-276 Bialystok, Poland \\ ${ }^{3}$ Department of Radiology, Medical University of Bialystok, 24A Maria Sklodowska-Curie Street, 15-276 Bialystok, Poland \\ ${ }^{4}$ Department of Gastroenterology and Internal Medicine, Medical University of Bialystok, 24A Maria Sklodowska-Curie Street, 15-276 Bialystok, Poland
}

\section{ARTICLEINFO}

Article history:

Received: 2 September, 2019

Accepted: 19 September, 2019

Published: 28 September, 2019

Keywords:

Negative esophagogastroduodenoscopy

overt upper gastrointestinal bleeding

hemodynamic instability

ruptured splenic artery aneurysm

arterial embolization

\begin{abstract}
A B S T R A C T
The manuscript presents a rare case of overt upper gastrointestinal bleeding initially manifesting as coffee ground vomiting, in a patient who had negative esophagogastroduodenoscopy performed a few hours before. Over the first 30 minutes in the Emergency Department, the patient developed an episode of hematemesis and hematochezia with hemodynamic instability. To identify the source of bleeding, contrastenhanced computed tomography was performed, which revealed a small aneurysm in the central part of the splenic artery with signs of intrapancreatic rupture. Re-esophagogastroduodenoscopy revealed active bleeding from the papilla of Vater and urgent selective angiography of the celiac trunk confirmed active extravasation of the contrast material into the main pancreatic duct. Successful embolization of the bleeding vessel was obtained by placing two coils in the aneurysm sac and the splenic artery.

The presented clinical case draws attention to a rare case of spontaneously ruptured splenic artery aneurysm causing visible only periodically hemosuccus pancreaticus, progressing quickly to a life-threatening condition and requiring firm and fast multi-profile treatment.
\end{abstract}

\section{Introduction}

Nonulcer, nonvariceal bleeding represents approximately $30 \%$ of all upper GI bleeding [1]. In $15 \%$ of patients, no source of hemorrhage can be identified, in $25 \%$ of cases bleeding do not cease spontaneously and require further intervention, sometimes a multidisciplinary approach, involving the interventional radiologist, gastroenterologist, and surgeon.

Case report

A 46-year-old alcoholic male was brought to a local hospital because of fatigue, abdominal pain, and coffee ground vomiting. Laboratory investigations showed a white blood cell count of $9.6 \times 10^{9} / \mathrm{L}$, a red blood cell count of $1.28 \times 10^{12} / \mathrm{L}$, a platelet count of $258 \times 10^{9} / \mathrm{L}$, a hemoglobin concentration of $30 \mathrm{~g} / \mathrm{L}$. Prothrombin time was within the normal range. He has transfused 3 units of packed red blood cells and the posttransfusion hemoglobin level remained 82 g/L. An esophagogastroduodenoscopy showed only superficial gastropathy without stigmata of bleeding. Then the patient was referred to the Department of Hepatology and was transported to the Medical University of Bialystok Clinical Hospital by ambulance.

On admission he was conscious, oriented, and had stable vitals, his blood

*Correspondence to: Adam R. Markowski, Department of Internal Medicine and Gastroenterology, Polish Red Cross Memorial Municipal Hospital, 79 Henryk Sienkiewicz Street, 15-003 Bialystok, Poland; Tel: +48-85-66-48-512; Fax: +48-85-66-48-512; E-mail: markowski@ szpitalpck.pl 
pressure was $123 / 75 \mathrm{mmHg}$, his heart rate was 86 beats/min, his respiratory rate was 16 breaths/min, a physical examination revealed pain on epigastric palpation, moderate ascites, and reduced breath sounds bilaterally consistent with a pleural effusion, a digital rectal examination showed no blood or masses. Over the next 30 minutes, the patient developed an episode of hematemesis and hematochezia with hypotension $86 / 52 \mathrm{mmHg}$ and tachycardia 102 beats/min. The patient was immediately referred to the Emergency Department with a presumptive diagnosis of severe gastrointestinal bleeding of unknown origin. He complained of epigastric pain and severe weakness. He did not take drugs prescribed by the doctor at the discharge from the hospital 3 months ago, he mentioned that he developed 2 weeks ago hematochezia for the very first time. He did not have a history of pancreatitis, abdominal surgery or drug-induced allergy. A physical examination showed moderate ascites with negative Blumberg sign.

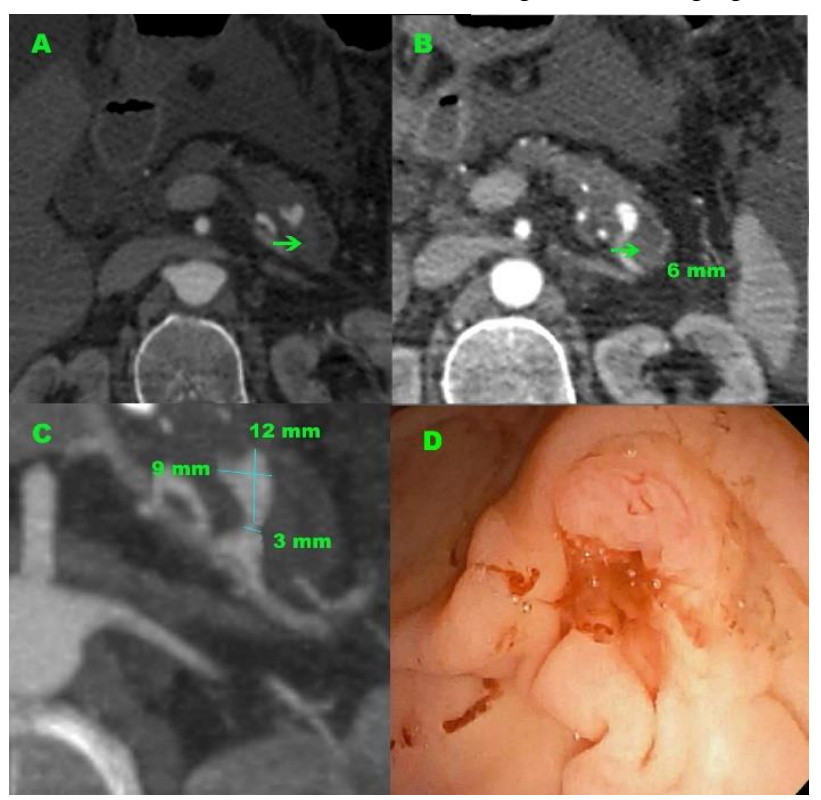

Figure 1: Abdominal axial CT images and endoscopic findings. (A) The saccular aneurysm of the splenic artery at the tail of the pancreas with presumed place of aneurysm rupture (arrow). (B) Dilated distal pancreatic duct (arrow) and a highly vascular nodular lesion at the pancreatic segment of the splenic artery was shown. (C) The splenic artery aneurysm (12 $\mathrm{mm}$ in size) was detected in the pancreas tail. (D) Duodenoscopy revealed active hemorrhage from the major papilla (arrow).

Laboratory investigations showed a white blood cell count of 8.74 $\times 10^{9} / \mathrm{L}$, a red blood cell count of $2.31 \times 10^{12} / \mathrm{L}$, a platelet count of 190 $\times 10^{9} / \mathrm{L}$, a hemoglobin concentration of $60 \mathrm{~g} / \mathrm{L}$, a hematocrit of $18.5 \%$, prothrombin time of 15.8 seconds. Initially, it was suspected the possibility of complications after esophagogastroduodenoscopy performed a few hours ago, and that is why the combined contrastenhanced thoracic and abdominal computed tomography was first performed to detect the presumptive perforation point and source of bleeding. CT revealed bilateral pleural effusion, centrally located filling defects in the pulmonary arteries of the lower lobes bilaterally corresponding to a pulmonary embolism, a large amount of non-dense fluid in the abdominal cavity and pelvis dependent on active alcoholic liver disease, a narrow pancreas with calcifications and features of chronic inflammation. Particular attention was paid to a aneurysm in the middle part of the splenic artery, directed to the front and surrounded by the parenchyma of the pancreatic tail (Figure 1abc). The aneurysm sac has a small size $(12 \mathrm{~mm} \times 9 \mathrm{~mm} \times 10 \mathrm{~mm})$ and a narrow neck $(3 \mathrm{~mm}$ wide) with a length of $4 \mathrm{~mm}$ and uneven and blurry contour suggesting the possibility of rupture and bleeding into the main pancreatic duct, which was segmentally widened in the tail $(6 \mathrm{~mm})$ and contained dense content (Figure 2).

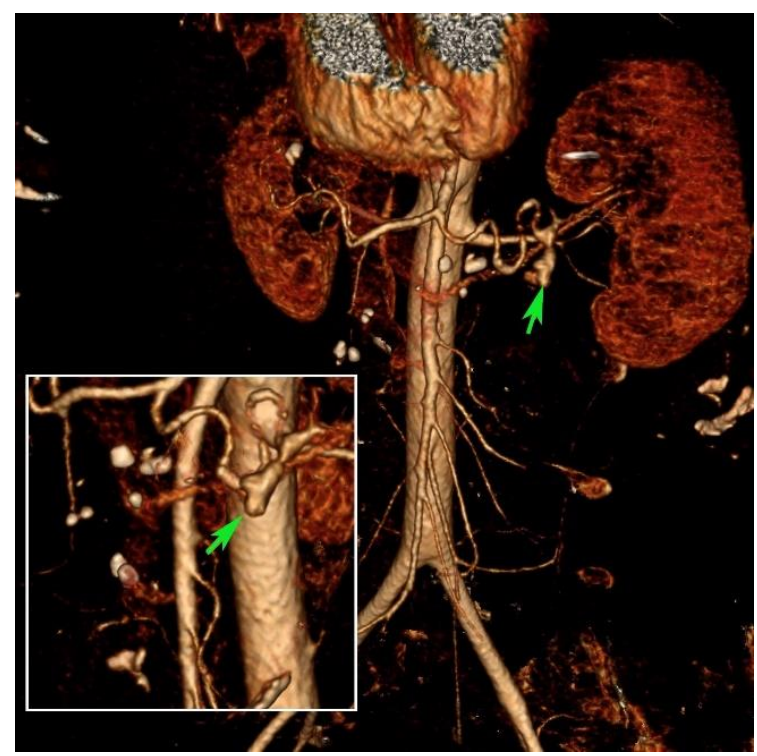

Figure 2: 3-dimensional CT images. A small bulge is visible (arrow) in the splenic artery aneurysm suggesting the presence of vessel perforation and the potential site of the arterio-pancreatic fistula.

The patient was managed with somatostatin infusion and blood transfusion, and he received 3 units of RBC \& 2 units of FFP. Somatostatin, rapid transfusions and fluid resuscitation resulted in hemodynamic stabilization and increase of hemoglobin level to $100 \mathrm{~g} / \mathrm{L}$. At that time, gastroscopy with forward-viewing endoscope detected fresh blood in the stomach and duodenum lumen, and the use of the sideviewing duodenoscope allowed for clear visualization of active bleeding from the ampulla of Vater (Figure 1d). Then, with a strong suspicion of a ruptured splenic artery aneurysm with secondary hemosuccus pancreaticus, the patient was referred for angiographic evaluation to Interventional Radiology Department to definitive confirms the diagnosis, bleeding visualization and location, and purpose of intravascular treatment.

Under local anesthesia, selective angiography of the celiac trunk was performed via a brachial approach and confirmed the presence of a splenic artery aneurysm, showed a point of wall rupture with active extravasation of contrast material from the arterial lumen and further pooling to form a small reservoir in the partially widened pancreatic duct (Figure 3). Pre-procedural knowledge of the hemorrhage site resulting in less delay. A decision was made to embolize the ruptured aneurysm using detachable coils. After catheterization of the splenic artery using a 5 French-size Multipurpose catheter, the successful embolization of the bleeding vessel was achieved with putting two detachable coils into the aneurysm sac and splenic artery lumen. Control arteriogram showed no flow distal to the coil mass and immediate collateral perfusion of the spleen from the other main arterial branches. The patient was transferred to a hospital ward. During further hospitalization, there was no 
recurrence of bleeding and the level of hemoglobin remained stable. After 6 days, the patient was discharged home.

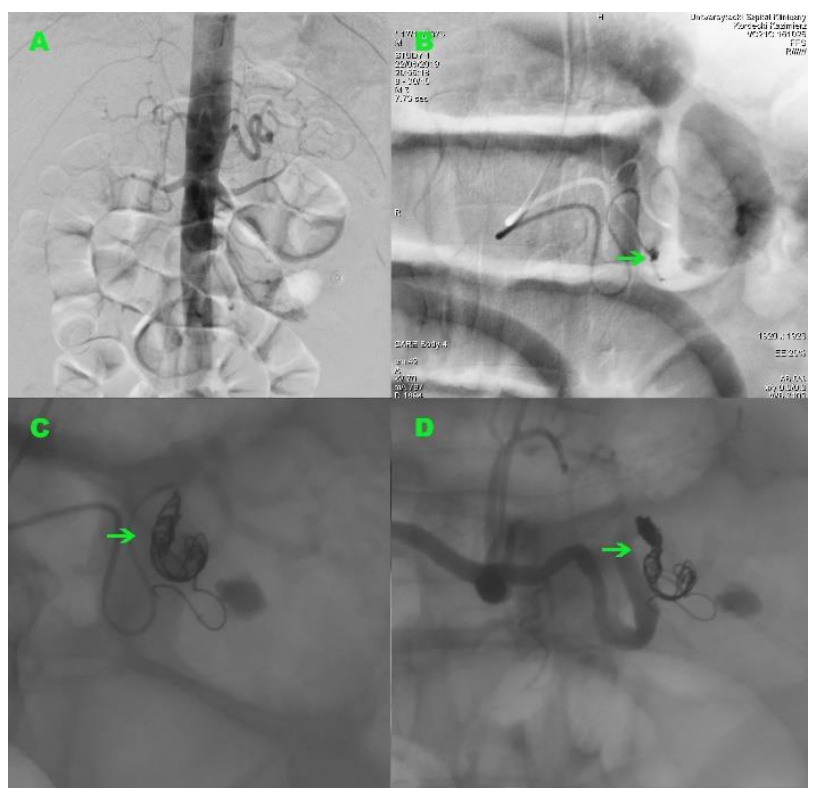

Figure 3: Angiographic findings. (A) Mapping aortography. Contrast extravasation is rarely visible, usually with massive bleeding rates (6 $\mathrm{mL} / \mathrm{min}$ ), pre-procedural knowledge of the hemorrhage site allowed for initial catheterization of a splenic artery resulting in less delay. (B) Selective celiac trunk injection shows the splenic artery aneurysm, the point of wall rupture with active extravasation of contrast material into the main pancreatic duct (arrow) and pooling to form a small reservoir. (C-D) The successful embolization of the bleeding vessel (arrow). Two coils placed in the aneurysm sac and in the lumen of the splenic artery.

\section{Discussion}

The reported incidence of all visceral arterial aneurysms (VAA) in autopsy is $2 \%$ and splenic artery aneurysms account for $60 \%[1,2] .22 \%$ of VAA present clinical emergency (leak or rupture) that have a mortality rate of $8.5 \%$ [2]. Spontaneously ruptured splenic artery aneurysm is a rare cause of hematemesis [2]. Hemosuccus pancreaticus (HP) is defined as bleeding from the ampulla of Vater via the pancreatic duct and is caused by a bleeding source in the pancreatic parenchyma, pancreatic duct or structures adjacent to the pancreas [3]. It seems that HP occurs five times more often in men than in women [3]. Most bleeding from the pancreatic duct is a consequence of hemorrhagic complications in the course of acute or chronic pancreatitis, resulting from injury of various surrounding arteries such as splenic, hepatic, gastric, gastroduodenal, or pancreaticoduodenal. Fewer common causes include aneurysms and arteriovenous malformations, ulcers of a pancreatic duct due to stones, pancreatic tumors and cysts, and additionally fibro-muscular dysplasia, atherosclerosis, infections, pancreatic trauma. Noteworthy are iatrogenic causes following diagnostic and therapeutic interventions within the pancreas such as fine needle aspiration biopsy or pancreatic duct stenting and drainage, during either conventional transpapillary ERCP or using EUS guidance. The bleeding is usually the occult or mild, intermittent and repetitive, leading in most patients only symptoms of chronic anemia, causing sometimes melena and hematemesis, rarely requiring rapid blood product transfusions and only sometimes causing hemodynamic instability and death. The intensity of bleeding, its natural course and outcome as well as the appearance of complications depends on the caliber of the injured blood vessel, on the general condition of the patient and co-existing diseases as well as the medications taken by the patient e.g. anticoagulation therapy. The colic pain may occur during the course of the bleeding as a result of the increased intraductal pressure caused by obstruction of the pancreatic duct due to clot formation.

Blood tests usually do not reveal significant findings except anemia or deficiency of clotting factors. The diagnosis is established on direct visualization of the hemorrhage through the main pancreatic duct at duodenoscopy or angiography. To exclude other, more frequent causes of bleeding, endoscopy is necessary. In some rare cases, active bleeding can be seen from the duodenal ampulla during duodenoscopy which is a cardinal finding and directs further diagnostics to the pancreas. However, HP is a rare cause of upper gastrointestinal bleeding (1/1500), and endoscopy can detect active bleeding from the papilla in only $30 \%$ of patients [3]. The break of aneurysm wall usually occurs as a sudden, single stage. Sometimes the rupture can occur in two stages resulting from the initial rupture being contained in the lesser sac before it spreads into the greater sac [4]. This course of the disease is responsible for 20$25 \%$ of all cases of splenic artery aneurysm rupture and usually presents as severe abdominal pain with a subsequent period of clinical stability collapse, followed by sudden collapse. The majority of the lesions are located in the distal third of the splenic artery, and some patients have multiple splenic aneurysms [5]. True aneurysms are treated based on their size, location, and symptoms, whereas all pseudoaneurysms require treatment due to high risk of rupture $(25 \%)$ and mortality $(50 \%)$, probably depending on the wall instability $[2,6]$.

Infrequently a dense content within the pancreatic duct or characteristic finding of clotted blood, known as the sentinel clot, may be observed on $\mathrm{CT}$, which suggests the bleeding from the pancreas. Computed tomography angiography (CTA) is a rapid and effective visualization technique at the early diagnostic stage but has its contraindications such as for example, kidney failure and allergy to contrast. CTA may show fuzzy outlines of the aneurysm boundaries, opacification of an aneurysmal artery and the presence of contrast outside the blood vessel. Direct catheter angiography (DCA) is a gold standard for ruptured aneurysm imaging causing hemosuccus pancreaticus, it has the highest resolution, detect the active bleeding, identifies the causative artery, confirms the presence of aneurysm, precisely identifies the rupture site and eventually, helps delineate arterial anatomy for further therapeutic interventions to quick stop bleeding. If a bleeding source is not identified provocative maneuvers may help identify the bleeding site without causing any hemodynamic instability [2].

Being the most sensitive technique during an acute episode and timeconsuming strategy, DCA is still a minimally invasive procedure, although it involves complications of arterial puncture (hematomas, pseudoaneurysms, hemorrhages, arterial dissection and occlusion, bacteremia and sepsis) and applied supplementary procedures (distal migration of embolic material, infarction, necrosis, abscess, perforation, contrast-induced nephropathy, postembolization syndrome). Angiography is especially indicated when the bleeding site cannot be identified by endoscopy or CT and bleeding cannot be controlled endoscopically. Eradicating the source of bleeding is the only way to cure HP and percutaneous arterial embolization could be the definitive 
treatment in any patient with severe bleeding in whom superselective catheterization is possible. If the source of bleeding can be found on angiography, interventional radiographic procedures are the first choice for treatment and have an overall success rate of $79-100 \%$ and recurrent bleeding rate $18-37 \%[3,7]$.

Endovascular management includes a few methods, however, the smaller the embolic agent, the bigger the likelihood of the end organ ischemia if the collateral circulation is insufficient [8]. Coil embolization is the most frequently described technique. It stimulates thrombosis in a aneurysm, which could successfully stop bleeding [3]. Application of stent grafts in an emergency situation may not be possible because of the tortuous anatomy of the vessel, small caliber size of the bleeding artery, as well as proximal and distal dimensional mismatch [9]. As arterial embolization is often a life-saving procedure, absolute contraindications do not exist, and relative contraindications include uncontrollable coagulopathy, renal insufficiency and contrast allergy [2]. In patients with hemodynamic instability or with failure of intravascular therapy, emergency surgical intervention is necessary. Pancreatic resection is needed in case of an unsuccessful ligation of the proximal bleeding vessel. Surgical procedures involve resection or ligature of bleeding vessels, and most studies show a success rate of $70-85 \%$, with a $20-25 \%$ mortality rate and $0-5 \%$ re-bleeding rate.

\section{Conclusions}

Bleeding from the upper gastrointestinal tract may be a life-threatening complication of digestive tract diseases. In some patients it is difficult to identify its source, then the intervention of a multidisciplinary team involving a gastroenterologist, interventional radiologist and sometimes a surgeon is necessary. Spontaneously ruptured splenic artery aneurysm is a very rare cause of bleeding from the papilla of Vater and even more rarely manifests as hematemesis, being an interesting clinical case, difficult to making accurate diagnosis.

\author{
Abbreviation \\ VAA: visceral arterial aneurysms. \\ FFP: fresh frozen plasma. \\ RBC: $\quad$ red blood cell. \\ CT: computed tomography. \\ CTA: computed tomography angiography. \\ HP: hemosuccus pancreaticus. \\ DCA: direct catheter angiography.
}

\section{Acknowledgements}

Not applicable.

\section{Authors' contributions}

All authors participated in the care of the patient as well as in acquisition, analysis, or interpretation data. ARM was responsible for coordinating treatment, acquiring and collecting data, searching the literature and drafting the manuscript. VG prepared and interpreted CT images. PR and $\mathrm{AB}$ performed esophagogastroduodenoscopy and duodenoscopy and took images. KK performed endovascular management and took images. All authors read, discussed the content and approved the final manuscript.

\section{Funding}

This research did not receive any specific grant from any funding agency in the public, commercial, or not-for-profit sectors.

\section{Ethics approval and consent to participate}

Not applicable.

\section{Consent for publication}

Not applicable. The identity of this patient has been protected.

\section{Conflicts of interest}

The authors declare that they have no competing interests.

\section{REFERENCES}

1. Chang MA, Savides TJ (2018) Endoscopic Management of Nonvariceal, Nonulcer Upper Gastrointestinal Bleeding. Gastrointest Endosc Clin N Am 28: 291-306. [Crossref]

2. Kandarpa K, Machan L, Durham J (2016) Handbook of Interventional Radiologic Procedures. Wolters Kluwer Health.

3. Yu P, Gong J (2018) Hemosuccus pancreaticus: A mini-review. Ann Med Surg (Lond) 28: 45-48. [Crossref]

4. Abhari P, Abhari S, Jackson A, Moustafa ASZ, Mercer L et al. (2019) Splenic Artery Aneurysm Case Report. Case Rep Obstet Gynecol 2019: 8347983. [Crossref]

5. Therakathu J, Panwala HK, Bhargava S, Eapen A, Keshava SN et al. (2018) Contrast-enhanced Computed Tomography Imaging of Splenic Artery Aneurysms and Pseudoaneurysms: A Single-center Experience. J Clin Imaging Sci 8: 37. [Crossref]

6. Branchi V, Meyer C, Verrel F, Kania A, Bölke E et al. (2019) Visceral artery aneurysms: evolving interdisciplinary management and future role of the abdominal surgeon. Eur J Med Res 24: 17. [Crossref]

7. Peynircioğlu B, Karaosmanoğlu AD, İdilman IS, Akata D, Şimşek H (2015) Intrapancreatic pseudoaneurysm causing massive gastrointestinal hemorrhage and chronic pancreatitis. Turk $J$ Gastroenterol 26: 270-273. [Crossref]

8. Lubarsky M, Ray C, Funaki B (2009) Embolization Agents - which One Should Be Used When? Part 1: Large-Vessel Embolization. Semin Intervent Radiol 26: 352-357. [Crossref]

9. Sul HR, Lee HW, Kim JW, Cha SJ, Choi YS et al. (2016) Endovascular management of hemosuccus pancreaticus, a rare case report of gastrointestinal bleeding. BMC Gastroenterol 16: 5. [Crossref] 